\title{
Laparoscopic First-stage Approach in a Two-stage Hepatectomy for Bilobar Colorectal Liver Metastases
}

\author{
Yuki Takahashi ${ }^{1}$, Kuniya Tanaka $^{2} \odot$, Tetsuji Wakabayshi ${ }^{3}$, Toshimitsu Shiozawa $^{4}$
}

\begin{abstract}
Aim: We reviewed a retrospectively collected database of 64 patients undergoing two-stage hepatectomy for colorectal liver metastases with special attention to cases involving a laparoscopic first stage.

Materials and methods: Three patients undergoing laparoscopic first-stage hepatectomy were analyzed and compared with 61 other patients who underwent two-stage hepatectomy using open surgery for the first stage.

Results: In three patients with a laparoscopic approach, the first-stage operation was a laparoscopic lateral sectionectomy or resection of segment 3, combined with portal vein embolization via the iliac vein directed at the contralateral hemiliver. No postoperative morbidity or mortality resulted. After a mean interval of 37.3 days, second-stage hepatectomy was performed for clearance of tumors in the right hemiliver (two in an open approach and one in a hybrid laparoscopic and open approach), with morbidity in $67 \%$ of patients (Clavien-Dindo classes I and IIIb in one patient each) but no mortality. When these three patients were compared with 61 patients treated with an open approach, numbers of metastatic tumors tended to be less in patients with a laparoscopic first stage. Duration of the first-stage hepatectomy $(p<0.01)$ and hospital stay after that hepatectomy were shorter in patients with laparoscopic resection than in patients with open resection ( $p=0.03$ ). Conclusion: Our preliminary data support the feasibility and safety of the laparoscopic approach for the first-stage resection during two-stage hepatectomy.

Clinical significance: First-stage laparoscopic clearance for patients with relatively small numbers of tumors who are anticipating two-stage hepatectomy for bilobar metastases becomes a standard option.
\end{abstract}

Keywords: Colorectal cancer, Laparoscopic resection, Liver metastases, Two-stage hepatectomy.

World Journal of Laparoscopic Surgery (2021): 10.5005/jp-journals-10033-1453

\section{INTRODUCTION}

Two-stage hepatectomy has emerged as a valuable strategy for curative treatment of patients with marginally resectable bilobar colorectal liver metastases that cannot be removed by a single hepatectomy without unacceptable risk of liver failure. At present, this procedure is performed routinely for patients with bilobar liver metastases from aggressive colorectal or neuroendocrine cancers at many hepatobiliary centers worldwide. Two-stage hepatectomy has improved resectability rates by 10 to $50 \%$ in unresectable or borderline-resectable patients, ${ }^{1-4}$ but this strategy risks considerable morbidity and high risk of disease progression after the first stage, leading to a reported drop-out rate of 15 to $30 \% .^{5}$

Ongoing experience with laparoscopic liver resection has gradually expanded indications for laparoscopic surgery to include major as well as minor hepatectomies. Although many reported case series have shown favorable results after open twostage hepatectomy, reports evaluating a laparoscopic approach to two-stage hepatectomy have remained limited, impeding discussion of a laparoscopic first stage in a two-stage hepatectomy. Laparoscopic resection for the first stage could reduce morbidity and possibly simplify the second operation by limiting adhesions. In fact, laparoscopy might allow one and possibly both stages to be performed with only minimal invasiveness.

Here, we report a small series of three patients undergoing a two-stage hepatectomy with a laparoscopic first-stage resection for colorectal liver metastases, providing some preliminary data regarding feasibility and safety.

\footnotetext{
${ }^{1-4}$ General and Gastroenterological Surgery, Showa University Fujigaoka Hospital, Yokohama, Kanagawa, Japan

Corresponding Author: Kuniya Tanaka, General and Gastroenterological Surgery, Showa University Fujigaoka Hospital, Yokohama, Kanagawa, Japan, Phone: +81-45-971-1151, e-mail: u1792ts235@med.showa-u.ac.jp
}

How to cite this article: Takahashi Y, Tanaka K, Wakabayshi T, et al. Laparoscopic First-stage Approach in a Two-stage Hepatectomy for Bilobar Colorectal Liver Metastases. World J Lap Surg 2021;14(2):90-94. Source of support: Nil

Conflict of interest: None

\section{Materials and Methods}

\section{Patients}

A database including 64 patients with colorectal liver metastases undergoing two-stage hepatectomy was reviewed. Among these patients, three had a laparoscopic first-stage hepatectomy. Here, we report details comparing these three patients to the 61 patients undergoing open two-stage hepatectomy. We further reviewed previously reported clinical series involving laparoscopic first stages in two-stage hepatectomy. The study protocol was approved by the ethics committee at our institutions (IRB protocol approval numbers, B110707040 and F2020C25). Written informed consent was obtained from all patients involved in this study. 


\section{Strategy for Hepatectomy}

Two-stage hepatectomy was indicated for advanced metastases requiring extensive liver resection. A prediction score ${ }^{6}$ of 50 or more, calculated using the formula $-84.6+0.933 a+1.11 b+0.999 c$ with a as the anticipated resection fraction (\%), b as the indocyanine green retention rate at 15 minutes (ICGR15, \%), and $c$ as patient age in years indicated treatment with a two-stage hepatectomy.

Most first procedures involved resection of metastases from the hemiliver intended to become the future liver remnant (FLR), followed by portal vein embolization (PVE) directed to the contralateral hemiliver. FLR volume was measured by computed tomography 3 weeks after the first hepatectomy. The second hepatectomy to resect the deportalized hemiliver typically was performed 4 weeks after the first procedure. When FLR volume was considered insufficient, completion surgery was postponed until sufficient FLR volume was attained or a smaller hepatectomy that initially planned was considered because of rapid tumor growth during the interval period.

\section{Laparoscopic Procedures}

The laparoscopic procedure began with the open insertion of an umbilical 12-mm port; five or six additional ports were used as well. Diagnostic laparoscopy was performed initially to confirm the absence of metastases in extrahepatic sites. Liver parenchymal transection was performed while maintaining a $12 \mathrm{~mm} \mathrm{Hg}$ pneumoperitoneum, which was increased to up to $20 \mathrm{~mm} \mathrm{Hg}$ if bleeding was encountered. Laparoscopic intraoperative ultrasonography was used routinely to guide resection and confirm resectability. Parenchymal transection was performed with a combination of a cavitron ultrasonic surgical aspirator system (Valley Lab, Boulder, Colorado) and a softcoagulation system (ERBE Elektromedizin, Tübingen, Germany). During parenchymal transection, Pringle's maneuver was performed to control vascular inflow, with 15 minutes of occlusion followed by 5 minutes of release. The resected specimen was placed in a plastic bag and retrieved through the umbilical incision after both cranial and caudal extensions.

PVE was attempted through the extended umbilical incision after retrieval of the specimen. The ileum was pulled out through the extended incision. For PVE, a 7-Fr catheter was inserted through an ileocolic vein, after which the portal branches of the hemiliver targeted for resection were embolized. The embolic material was a mixture of gelatin pellets (Gelfoam powder; Upjohn, Kalamazoo, Michigan) and oleic acid monoethanolamine (Oldamine; Grelan, Tokyo, Japan). After restaging following PVE, patients were suitably scheduled for a second-stage resection to remove tumors from the remnant liver.

Our standard approach at the second-stage hepatectomy following a laparoscopic first hepatectomy is a hybrid of laparoscopic and open approaches. Generally, mobilization of the right hemiliver is performed laparoscopically, and transection of liver parenchyma is performed under minimum laparotomy as previously reported. ${ }^{7}$ Planning for the second procedure must be flexible, with minimization when FLR hypertrophy is suboptimal. Multiple small resections avoiding excessive tumor-free margins are performed using an open approach.

\section{Terminology and Analyzed Parameters}

The Brisbane 2000 terminology of the International HepatoPancreato-Biliary Association was used to designate operative procedures. ${ }^{8}$ Morbidities were assessed according to the ClavienDindo (CD) classification. ${ }^{9}$

\section{Statistical Analysis}

Continuous variables, analyzed using the Mann-Whitney $U$ test, are expressed as the mean \pm standard deviation. Categorical variables, expressed as numbers followed by percentages in parentheses, were analyzed with Fisher's exact test. A difference was considered significant when the two-sided $p$-value was below 0.05 . All statistical analyses were carried out using SPSS statistical software (version 23; IBC SPSS, Chicago, Illinois).

\section{Results}

Details of three patients with laparoscopic two-stage hepatectomy (two men, and one woman; mean age, $67.0 \pm 7.2$ years) are shown in Table 1. The first-stage hepatectomy consisted of laparoscopic lateral sectionectomy or resection of segment 3. All three patients underwent PVE to the contralateral hemiliver via the iliac vein during the first-stage laparoscopic hepatectomy. Second-stage hepatectomy was performed after a mean interval of $37.3 \pm 10.7$ days following first-stage resection. Adhesions were considered minimal on assessment during the second-stage procedure in all patients. Metastatic tumors were removed from the right hemiliver at secondstage hepatectomy using an open approach in two patients and a hybrid laparoscopic and open approach in the other patient. In the two patients undergoing open second-stage hepatectomy, part of the deportalized hemiliver was left in place because remnant liver hypertrophy and liver function were compromised by prehepatectomy chemotherapy. We resected segment 8 and performed multiple partial hepatectomies in one patient. Another underwent resection of segment 7 extending to 8 in addition to resection of the right hepatic vein (with preservation of the right inferior hepatic vein) and partial resection of segments 5 and 6 . The third patient was treated with a hybrid approach including posterior sectionectomy extended to segment 8 with preservation of the right

Table 1: Characteristics and operative feasibility of patients undergoing two-stage hepatectomy

\begin{tabular}{|c|c|c|c|c|c|c|c|c|c|c|}
\hline & & No. of & Maximum & & Procedures & $\begin{array}{l}\text { Resected } \\
\text { volume, gm }\end{array}$ & $\begin{array}{l}\text { Duration, } \\
\text { minute }\end{array}$ & $\begin{array}{l}\text { Blood loss, } \\
\mathrm{mL}\end{array}$ & Morbidity, \% & $\begin{array}{l}\text { Hospital } \\
\text { stay, days }\end{array}$ \\
\hline & Gender & tumors & diameter, $\mathrm{mm}$ & $P V E$ & First/second & First/second & First/second & First/second & First/second & First/second \\
\hline 1 & Male & 11 & 35 & Performed & $\begin{array}{l}\text { Lateral section/ } \\
\text { segment } 8+\mathrm{P}\end{array}$ & $175 / 223$ & $230 / 374$ & $500 / 700$ & None/none & $8 / 10$ \\
\hline 2 & Female & 6 & 40 & Performed & $\begin{array}{l}\text { Lateral section/ } \\
\text { Ext. posterior } \\
\text { section }\end{array}$ & $190 / 317$ & $255 / 455$ & $378 / 700$ & None/CD-I & $5 / 14$ \\
\hline 3 & Male & 5 & 33 & Performed & $\begin{array}{l}\text { Segment 3/Ext. } \\
\text { segment } 7+P\end{array}$ & $54 / 264$ & $238 / 559$ & $380 / 635$ & None/CD-IIIb & $9 / 31$ \\
\hline
\end{tabular}

No., number; PVE, portal vein embolization; first, first hepatectomy; second, second hepatectomy; section, sectionectomy; segment, segmentectomy; P, partial hepatectomy; Ext., extended to; CD, Clavien-Dindo 
hepatic vein because insufficient FLR hypertrophy precluded right hemihepatectomy.

No morbidity or mortality followed the first-stage liver resection. The second-stage resection was associated with no mortality, but two of three patients experienced operative morbidity. Complications after the second resection included a prolonged inflammatory state of unknown cause requiring antibiotic administration (CD class I) in one patient and postoperative bleeding requiring surgical intervention (CD class IIlb) in another.

When these three patients were compared with 61 who underwent an open first stage, small numbers precluded statistical significance. Metastatic tumors tended to be fewer in the patients undergoing laparoscopic surgery. Although hepatectomy procedures differed between the laparoscopic and the open group at both firstsecond-stage hepatectomy ( $p<0.001$ and $p=0.013$, respectively), duration of the first-stage hepatectomy $(p<0.01)$ and hospital stay after the first-stage hepatectomy $(p=0.03)$ were shorter in patients with laparoscopic resection than open resection. Total resected volume at second-stage hepatectomy was smaller in the laparoscopic group than in the open group $(p=0.016)$ because the procedures in the laparoscopic group had to be minimized because of insufficient remnant liver volume and functional hypertrophy (Table 2).

Table 2: Comparison of two-stage hepatectomies between laparoscopic and open approaches

\begin{tabular}{|c|c|c|c|c|}
\hline & & $\begin{array}{l}\text { Laparoscopic } \\
(n=3)\end{array}$ & $\begin{array}{l}\text { Open } \\
(n=61)\end{array}$ & pvalue \\
\hline Age, years & & $67.0 \pm 7.2$ & $61.5 \pm 10.5$ & 0.409 \\
\hline \multirow[t]{2}{*}{ Gender } & Male & $2(67 \%)$ & $36(59 \%)$ & $>0.999$ \\
\hline & Female & $1(33 \%)$ & $25(41 \%)$ & \\
\hline \multicolumn{5}{|l|}{ Timing of metastases relative to primary } \\
\hline & Synchronous & $3(100 \%)$ & $54(89 \%)$ & $>0.999$ \\
\hline & Metachronous & 0 & $7(11 \%)$ & \\
\hline Tumor number & & $7.3 \pm 3.2$ & $13.1 \pm 8.5$ & 0.214 \\
\hline Maximum tumor size, $\mathrm{mm}$ & & $36.0 \pm 3.6$ & $53.3 \pm 35.9$ & 0.583 \\
\hline Extrahepatic metastases present & & $2(67 \%)$ & $15(25 \%)$ & 0.170 \\
\hline Preoperative serum CEA, ng/mL & & $14.1 \pm 4.1$ & $400.4 \pm 1446.2$ & 0.651 \\
\hline \multicolumn{5}{|l|}{ Prehepatectomy chemotherapy } \\
\hline & Performed & $3(100 \%)$ & $52(85 \%)$ & $>0.999$ \\
\hline \multicolumn{5}{|l|}{ First hepatectomy } \\
\hline \multirow[t]{5}{*}{ Extent of resection } & Partial & 0 & $14(23 \%)$ & $<0.001$ \\
\hline & Multiple partial & 0 & $34(56 \%)$ & \\
\hline & Segment or more & $1(33 \%)$ & 0 & \\
\hline & Section or more & $2(67 \%)$ & $10(16 \%)$ & \\
\hline & Hemiliver & 0 & $3(5 \%)$ & \\
\hline Duration, min & & $241 \pm 12.8$ & $423.6 \pm 112.2$ & 0008 \\
\hline Bleeding, $\mathrm{mL}$ & & $419.3 \pm 69.9$ & $722.0 \pm 848.8$ & 0.906 \\
\hline Resected volume, gm & & $139.7 \pm 74.6$ & $155.5 \pm 200.3$ & 0.537 \\
\hline Morbidity, \% & & 0 & $21(33 \%)$ & 0.545 \\
\hline Hospital stay, days & & $7.3 \pm 2.1$ & $18.6 \pm 11.8$ & 0.029 \\
\hline Portal vein embolization performed & $3(100 \%)$ & $52(85 \%)$ & 0.999 & \\
\hline Interval, days & $37.3 \pm 10.7$ & $72.1 \pm 60.2$ & 0.263 & \\
\hline \multicolumn{5}{|l|}{ Second hepatectomy } \\
\hline \multirow[t]{7}{*}{ Extent of resection } & Multiple partial & 0 & $3(6 \%)$ & 0.013 \\
\hline & Segment or more & $2(67 \%)$ & $2(4 \%)$ & \\
\hline & Section or more & $1(33 \%)$ & $4(8 \%)$ & \\
\hline & Hemiliver & 0 & $20(38 \%)$ & \\
\hline & Bisections or more & 0 & $2(4 \%)$ & \\
\hline & Extended hemiliver & 0 & $18(35 \%)$ & \\
\hline & Trisections & 0 & $3(6 \%)$ & \\
\hline Duration, min & & $462.7 \pm 92.7$ & $471.2 \pm 147.2$ & 0.699 \\
\hline Bleeding, $\mathrm{mL}$ & & $578.3 \pm 157.8$ & $1592.6 \pm 1728.9$ & 0.152 \\
\hline Resected volume, gm & & $268.0 \pm 47.1$ & $567.6 \pm 263.3$ & 0.016 \\
\hline Morbidity, \% & & $2(67 \%)$ & $17(33 \%)$ & 0.555 \\
\hline Hospital stay, days & & $18.3 \pm 11.2$ & $25.5 \pm 18.9$ & 0.548 \\
\hline Mortality, \% & & 0 & $1(2 \%)$ & $>0.999$ \\
\hline
\end{tabular}

CEA, carcinoembryonic antigen. Continuous data are expressed as the mean \pm standard deviation 


\section{Discussion}

In two-stage hepatectomy, complication rates have varied from 0 to $30 \%^{2,10,11}$ for the first stage and ranged up to $60 \%^{1}$ for the second. Higher complication rates after second-stage surgery are widely acknowledged and likely are related to prolonged prehepatectomy chemotherapy, complicated surgical procedures, and massive volumes of liver resection. ${ }^{12}$ Advantages of laparoscopic approach to liver resection have been well described, including less postoperative pain, fewer intra-abdominal adhesions, and shorter hospital stays. ${ }^{13-15}$ Recently, laparoscopic approaches are gradually being applied to two-stage hepatectomy, ${ }_{1}^{16-20}$ offering the benefit of less invasiveness. However, overall surgical feasibility of twostage hepatectomy using a laparoscopic approach remained an ongoing concern.

In this study, the total number of metastases tended to be smaller in patients undergoing the laparoscopic approach than in those treated with an open approach. However, as expected, laparoscopy decreased length of the operation and the hospital stay and was associated with somewhat fewer postoperative complications after first-stage hepatectomy. The laparoscopic firststage approach provoked fewer adhesions, which should facilitate the second stage.

Generally, inflammation of the portal pedicle after PVE is associated with dense abdominal and perihepatic adhesions, and anatomy is distorted by liver hypertrophy following the previous resection. As a result, laparoscopic second-stage hepatectomy can be technically challenging, requiring exceptional expertise in both laparoscopic maneuvers and hepatobiliary surgery. A hybrid procedure combining laparoscopic and open approaches for the second hepatectomy is the least invasive strategy that we now can apply. Unfortunately, multiple small resections within the deportalized liver in lieu of major hepatectomy via an open approach were required in two patients with insufficient functional hypertrophy according to liver function parameters compromised by perioperative chemotherapy. The other patient could not tolerate right hemihepatectomy, so we performed posterior sectionectomy extended to segment 8 using a hybrid approach. In spite of these limitations, our short-term outcome was comparable or slightly better in terms of intraoperative bleeding and duration of hospital stay than the same measures in 61 patients with an open approach.

According to previous reports regarding laparoscopic two-stage hepatectomy (Table 3), laparoscopic second-stage hepatectomy was completed in 58 of 82 patients $(70.7 \%)$. This high completion rate for laparoscopic second resection could be explained by the restriction of some studies to patients eligible for laparoscopic resection at both stages and also by stringent criteria, including a limited number of liver metastases. The mean or median total number of metastatic tumors was about 5 in these reported series; such a small number of metastases might have been managed with only a single hepatectomy in some instances. Further, the mortality rate in two reports in Table $3^{18,20}$ with a high completion rate for laparoscopic resections in both stages was about 3\%, which is similar to or slightly greater than mortality in open two-stage hepatectomy. 2,10,11,20 Based on these results, laparoscopic second-stage resection should be limited to patients with relatively few remaining metastases. General application of laparoscopic resection to both stages now remains an elusive goal.

Given our small numbers of patients, long-term results would be difficult to generalize. However, at this writing, all three patients remain alive at 90,445 , and 1,345 postoperative days. Some controversy exists regarding the risk of compromising oncologic principles when a minimally invasive approach is adopted. However, recently reported long-term results for patients with laparoscopic two-stage hepatectomy were comparable to results for open twostage hepatectomy. ${ }^{20}$ A laparoscopic approach might not adversely affect the oncologic course of patients with two-stage hepatectomy for bilobar colorectal liver metastases.

\section{ConCLUSION}

Our preliminary data support the feasibility and safety of the laparoscopic approach for first-stage liver resection. Advantages of first-stage laparoscopic hepatectomy include fewer adhesions and rapid postoperative recovery. This approach should be offered to patients with relatively small numbers of tumors who

Table 3: Reported series of laparoscopic two-stage hepatectomy

\begin{tabular}{|c|c|c|c|c|c|c|c|c|c|}
\hline \multirow[b]{2}{*}{ Authors } & \multirow{2}{*}{$\begin{array}{l}\text { No. of } \\
\text { patients } \\
\text { First/ } \\
\text { Second }\end{array}$} & \multirow[b]{2}{*}{$\begin{array}{l}\text { No. of } \\
\text { tumors }\end{array}$} & \multirow[b]{2}{*}{$\begin{array}{l}\text { PVE } \\
\text { performed }\end{array}$} & \multirow{2}{*}{$\begin{array}{l}\text { Approach (pure/ } \\
\text { conversion/open) } \\
\text { First/ } \\
\text { Second }\end{array}$} & \multirow{2}{*}{$\begin{array}{l}\text { Duration, minute } \\
\text { First/ } \\
\text { Second }\end{array}$} & \multirow{2}{*}{$\begin{array}{l}\text { Blood loss, } \mathrm{mL} \\
\text { First/ } \\
\text { Second }\end{array}$} & \multirow{2}{*}{$\begin{array}{l}\text { Morbidity, } \\
\% \\
\text { First/ } \\
\text { Second }\end{array}$} & \multirow{2}{*}{$\begin{array}{l}\text { Hospital stay, } \\
\text { days } \\
\text { First/ } \\
\text { Second }\end{array}$} & \multirow{2}{*}{$\begin{array}{l}\text { Overall } \\
\text { mortality, } \\
\%\end{array}$} \\
\hline & & & & & & & & & \\
\hline \multirow[t]{2}{*}{ Di Fabio $^{16}$} & $8 / 8$ & $4(2-6)$ & 7 & $8 / 0 / 0$ & $139 \pm 45 /$ & $132 \pm 103 /$ & $0 / 50$ & $6(4-10) /$ & 0 \\
\hline & & & & $2 / 1 / 5$ & $243 \pm 85$ & $1,225 \pm 468$ & & $15.5(6-43)$ & \\
\hline \multirow[t]{2}{*}{ Sandri ${ }^{17}$} & $4 / 4$ & & 4 & $4 / 0 / 0$ & $189 / 304$ & $22 / 425$ & 0 & $3.5 / 8$ & 0 \\
\hline & & & & $0 / 0 / 4$ & & & - & & \\
\hline \multirow[t]{2}{*}{ Fuks $^{18}$} & $34 / 26$ & $6.0 \pm 7.1$ & 20 & $32 / 2 / 0$ & $210 \pm 114 /$ & $150 \pm 143 /$ & $50 / 54$ & $6.1 \pm 5.2 /$ & 3 \\
\hline & & & & $22 / 4 / 0$ & $250 \pm 139$ & $250 \pm 203$ & & $9 \pm 8.2$ & \\
\hline \multirow[t]{2}{*}{ Kilburn ${ }^{19}$} & $7 / 6$ & $4(3-10)$ & 7 & $7 / 0 / 0$ & $100(60-170) /$ & $100(50-400) /$ & $0 / 50$ & $3(2-5) /$ & 0 \\
\hline & & & & $0 / 1 / 5$ & $158(120-220)$ & $420(100-600)$ & & $6.5(5-23)$ & \\
\hline \multirow[t]{2}{*}{ Okumura ${ }^{20}$} & $38 / 38$ & $6(2-13)$ & 25 & $37 / 1 / 0$ & $159(70-415) /$ & $50(0-350) /$ & $16 / 26$ & $6(0-34) /$ & 2.6 \\
\hline & & & & $34 / 4 / 0$ & $305(150-480)$ & $225(50-1,300)$ & & $9(4-49)$ & \\
\hline
\end{tabular}

No., number; PVE, portal vein embolization; pure, pure laparoscopic; conversion, conversion from laparoscopic to open surgery; open, open-abdomen; first, first hepatectomy; second, second hepatectomy. Data are expressed as the mean \pm standard deviation or the median followed by range in parentheses 
are anticipating two-stage hepatectomy for bilobar metastases. With time, first-stage laparoscopic clearance of the left hemiliver becomes a standard option.

\section{Clinical Significance}

First-stage laparoscopic clearance for patients with relatively small numbers of tumors who are anticipating two-stage hepatectomy for bilobar metastases becomes a standard option.

\section{OrCID}

Kuniya Tanaka 자 https://orcid.org/0000-0002-3871-8208

\section{References}

1. Adam R, Laurent A, Azoulay D, et al. Two-stage hepatectomy: a planned strategy to treat irresectable liver tumors. Ann Surg 2000;232(6):777-785. DOI: 10.1097/00000658-200012000-00006.

2. Jaeck D, Oussoultzoglou E, Rosso E, et al. A two-stage hepatectomy procedure combined with portal vein embolization to achieve curative resection for initially unresectable multiple and bilobar colorectal liver metastases. Ann Surg 2004;240(6):1037-1049. DOI: 10.1097/01.sla.0000145965.86383.89.

3. Chun YS, Vauthey JN, Ribero D, et al. Systemic chemotherapy and two-stage hepatectomy for extensive bilateral colorectal liver metastases: perioperative safety and survival. J Gastrointest Surg 2007;11(11):1498-1504. DOI: 10.1007/s11605-007-0272-2.

4. de Haas RJ, Wicherts DA, Flores E, et al. R1 resection by necessity for colorectal liver metastases: is it still a contraindication to surgery? Ann Surg 2008;248(4):626-637. DOI: 10.1097/SLA.0b013e31818a07f1.

5. Wicherts DA, Miller R, de Haas RJ, et al. Long-term results of two-stage hepatectomy for irresectable colorectal cancer liver metastases. Ann Surg 2008;248(6):994-1005. DOI: 10.1097/SLA.0b013e3181907fd9.

6. Yamanaka N, Okamoto E, Oriyama T, et al. A prediction scoring system to select the surgical treatment of liver cancer. Further refinement based on 10 years of use. Ann Surg 1994;219(4):342-346. DOI: 10.1097/00000658-199404000-00003.

7. Nitta $H$, Sasaki A, Fujita T, et al. Laparoscopy-assisted major liver resections employing a hanging technique: the original procedure. Ann Surg 2010;251(3):450-453. DOI: 10.1097/SLA.0b013e3181cf87da.

8. Terminology Committee of the International Hepato-PancreatoBiliary Association. The Brisbane 2000 terminology of liver anatomy and resection. HPB 2000;2(3):333-339. DOI: 10.1016/S1365$182 X(17) 30755-4$.
9. Dindo D, Demartines N, Clavien PA. Classification of surgical complications: a new proposal with evaluation in a cohort of 6336 patients and results of a survey. Ann Surg 2004;240(2):205-213. DOI: 10.1097/01.sla.0000133083.54934.ae.

10. Pamecha V, Nedjat-Shokouhi B, Gurusamy K, et al. Prospective evaluation of two-stage hepatectomy combined with selective portal vein embolisation and systemic chemotherapy for patients with unresectable bilobar colorectal liver metastases. Dig Surg 2008;25(5):387-393. DOI: 10.1159/000176063.

11. Tanaka K, Matsuo K, Murakami T, et al. Associating liver partition and portal vein ligation for staged hepatectomy (ALPPS): short-term outcome, functional changes in the future liver remnant, and tumor growth activity. Eur J Surg Oncol 2015;41(4):506-512. DOI: 10.1016/ j.ejso.2015.01.031.

12. Karoui M, Penna C, Amin-Hashem M, et al. Influence of preoperative chemotherapy on the risk of major hepatectomy for colorectal liver metastases. Ann Surg 2006;243(1):1-7. DOI: 10.1097/01. sla.0000193603.26265.c3.

13. Tranchart H, Dagher I. Laparoscopic liver resection: a review. J Visc Surg 2014;151(2):107-115. DOI: 10.1016/j.jviscsurg.2013.10.003.

14. Zhou $Y, X i a o ~ Y, W u ~ L$, et al. Laparoscopic liver resection as a safe and efficacious alternative to open resection for colorectal liver metastasis: a meta-analysis. BMC Surg 2013;13:44. DOI: 10.1186/14712482-13-44.

15. Mirnezami R, Mirnezami AH, Chandrakumaran K, et al. Short- and long-term outcomes after laparoscopic and open hepatic resection: systematic review and meta-analysis. HPB (Oxford) 2011;13(5): 295-308. DOI: 10.1111/j.1477-2574.2011.00295.x.

16. Di Fabio F, Whistance R, Rahman S, et al. Exploring the role of laparoscopic surgery in two-stage hepatectomy for bilobar colorectal liver metastases. J Laparoendosc Adv Surg Tech A 2012;22(7):647-650. DOI: 10.1089/lap.2012.0163.

17. Levi Sandri GB, Colace L, Vennarecci G, et al. Laparoscopic first step approach in the two stage hepatectomy. Hepatobiliary Surg Nutr 2015;4(5):345-347. DOI: 10.3978/j.issn.2304-3881.2015.01.13.

18. Fuks D, Nomi T, Ogiso S, et al. Laparoscopic two-stage hepatectomy for bilobar colorectal liver metastases. Br J Surg 2015;102(13):16841690. DOI: 10.1002/bjs.9945.

19. Kilburn DJ, Chiow AK, Lewin J, et al. Laparoscopic approach to a planned two-stage hepatectomy for bilobar colorectal liver metastases. ANZ J Surg 2016;86(10):811-815. DOI: 10.1111/ ans.12748.

20. Okumura S, Goumard C, Gayet B, et al. Laparoscopic versus open two-stage hepatectomy for bilobar colorectal liver metastases: a bi-institutional, propensity score-matched study. Surgery 2019;166(6):959-966. DOI: 10.1016/j.surg.2019.06.019. 